\title{
¿Reformar sin los docentes?: identidad profesional, resistencia, y mediación a la reforma de la educación secundaria en Perú
}

\section{Angela Bravo Chacón}

https://orcid.org/0000-0003-1191-5727

angelabravo.c@gmail.com

Recibido: 06/01/2021

Aprobado: 29/05/2021 


\section{¿Reformar sin los docentes?: identidad profesional, resistencia, y mediación a la reforma de la educación secundaria en Perú ${ }^{1}$}

\section{Resumen}

¿Puede una reforma educativa ignorar la agencia y mediación de los docentes? ¿Cómo se sentirán los profesionales docentes al ser asumidos como ejecutores de programas diseñados por técnicos? Este artículo analiza las respuestas e imaginarios de docentes rurales sobre su profesionalismo, y explora la mediación y resistencia ejercidas hacia la política de sesiones estandarizadas diseñada por el Ministerio de Educación para la implementación de la Jornada Escolar Completa (JEC). El caso proporciona un ejemplo de libro de texto de una reforma jerárquica basada en los enfoques neoliberales de diseño único y accountability vertical, implementada en un contexto de duras condiciones laborales y desprofesionalización del rol docente.

Este artículo está basado en una investigación exploratoria en dos escuelas secundarias rurales. Los hallazgos muestran que, en el contexto de la implementación de sesiones estandarizadas JEC, el rol profesional docente es retado y se reconfigura en todas sus dimensiones: juicio profesional, ejercicio de identidad, autovaloración y postura política. Los docentes reaccionan al desafío en su agencia y autonomía profesional mediante la construcción de respuestas contradictorias hacia la reforma y las acciones de resistencia a la implementación impulsadas por un discurso cargado de imperativos competitivos y contradictorios. Además, los hallazgos muestran que, en las escuelas rurales JEC, el rechazo a la reforma y los puntos débiles del diseño de política son usados para reforzar la utilización de estrategias de autonomía profesional negativas, como justificar absentismo, al tiempo que aumenta la alienación, resistencia y desconfianza hacia la propia noción de reforma.

Palabras clave: Accountability educacional, autonomía profesional docente, profesionales de la educación, reforma educativa

1. El presente artículo recoge la investigación realizada para la tesis de grado en maestría en Ciencias Sociales (Msc) de la Universidad de Glasgow, Reino Unido. 


\title{
Reforming Without the Teachers? Professional Identity, Resistances, And Mediation to the Secondary Education Reform in Peru
}

\begin{abstract}
Can an educational reform ignore the agency and mediation of teachers? How does it feel for teachers' professionalism to be assumed as executors of programs designed by external policy makers? Under these premises, this article analyzes the responses and ideas about professionalism, and explores the mediation and resistance of secondary school teachers towards the standardized sessions policy designed by Ministry of Education in the context of the implementation of the Full School Day (JEC). The case provides a textbook example of a hierarchical reform based on neoliberal single-design and accountability approaches to fidelity of implementation in a particular context of harsh working conditions and deprofessionalization of secondary school teachers.

This article is based on an exploratory research product of in-depth field work in two rural secondary schools. Findings show that, in the context of the JEC reform, the professional teaching role is reconfigured in all its dimensions: professional judgment, exercise of identity and self-assessment, and political positions. Teachers react to this challenge in their professional agency by constructing contradictory responses to reform and actions of resistance to implementation driven by competitive imperatives in their discourse. In addition, findings reveal that, in the context of rural JEC schools, given the conditions of high inequality and challenge, the rejection of the reform and its weaknesses are ways of reinforcing the use of negative professional strategies, such as absenteeism, alienation, and mistrust towards those policies proposed by MINEDU.
\end{abstract}

Keywords: Educational accountability, teachers' professional authonomy, education professionals, educational reform 


\section{Introducción}

El bajo desempeño de Perú en PISA $2012^{2}$ generó una fuerte presión pública sobre la calidad de la educación secundaria y aceleró la respuesta del Ministerio de Educación (Minedu) para iniciar una reforma en este nivel de la Educación Básica. En ese contexto, en julio de 2014, se ordenó la creación de un comité integrado por nueve funcionarios del sector, quienes contaban con un plazo de solo veinticinco días para finalizar un diseño de política para mejorar la calidad de la educación secundaria y treinta días adicionales para presentar un plan de implementación para la reforma ${ }^{3}$.

El comité aprobó una serie de modelos de servicio educativo para diversificar la secundaria, y mejorar la calidad y pertinencia del servicio en las secundarias públicas. Uno de estos modelos inició una ambiciosa política educativa denominada Jornada Escolar Completa (JEC) ${ }^{4}$. El modelo fue aprobado en setiembre de 2014 e inició su implementación en marzo de 2015; a partir de este, se impulsó la extensión de la jornada escolar de 35 a 45 horas pedagógicas semanales. Además de la extensión horaria, se ofrecieron mejores condiciones de equipamiento, recursos humanos, y una reforma de la gestión y el trabajo pedagógico en mil escuelas secundarias seleccionadas por el propio Minedu.

Aunque el diseño mismo de la reforma JEC presenta un ejemplo excepcional de una política centralizada y un diseño de reforma jerárquica, un aspecto de esta es particularmente interesante de analizar. En los primeros años de la reforma, el Minedu decidió implementar el componente pedagógico a través de la entrega de sesiones estandarizadas. Estas sesiones estuvieron diseñadas a modo de guiones exhaustivos que indicaban a los docentes cómo conducir una sesión de clase significativa y cercana al enfoque por competencias. La decisión fue tomada dados los cortos plazos de implementación, los que impedían apostar por una reforma pedagógica más profunda basada en la transformación de prácticas docentes. Asimismo, responde a la necesidad de controlar las prácticas de las escuelas, bajo el supuesto general del fracaso de la autonomía

2. Perú había participado previamente en rondas de PISA; sin embargo, la ronda del año 2013 era la primera en la que el país aparecería en el ranking de resultados publicado por la OCDE comparando el desempeño de los adolescentes de 15 años en competencias de lectura, matemática y ciencia. En esta ocasión, el Perú ocupó último lugar del ranking publicado por la OCDE.

3. Se trata de la "Resolución Ministerial N 301-2014-MINEDU: Creación del Grupo de Trabajo, encargado de formular el modelo de servicio educativo: Jornada Escolar Completa para las instituciones educativas públicas del nivel de educación secundaria, el plan de implementación y las acciones de seguimiento del referido modelo".

4. Cabe mencionar que el modelo de reforma de la secundaria Jornada Escolar Completa adapta para el Perú políticas similares impulsadas en Chile y Colombia. 
docente que suele acompañar las reformas reactivas a los resultados de PISA (Bolívar, 2011; Hult y Edström, 2016)5.

Inicialmente, las orientaciones para la implementación de las sesiones elaboradas por el Minedu señalaban que los docentes debían adaptarlas, puesto que se trataban de ejemplos sobre cómo desarrollar aprendizajes activos y en el marco del enfoque por competencias. Sin embargo, en los siguientes meses, el objetivo de las sesiones cambió, lo que generó poca claridad sobre si los docentes debían usarlas solo como ejemplos a adaptar o si debían aplicarlas de manera estandarizada.

Este cambio en el sentido en el uso de las sesiones responde a dos acciones tomadas por el Minedu. Primero, las sesiones pasaron de ser ejemplos a ser paquetes mensuales de unidades didácticas, alineados a los textos escolares estandarizados, los que fueron reemplazando la carpeta pedagógica anual que diseñan los docentes. Segundo, una vez incluidos en las carpetas pedagógicas, el uso en aula de las sesiones estandarizadas fue incorporado en los sistemas de seguimiento a la implementación del componente pedagógico de la reforma JEC. Así, se aplicó un sistema agresivo de accountability ${ }^{6}$ vertical, centrado en evaluaciones, semáforos y visitas de observación inopinada.

En este contexto, este artículo explora, desde el marco de la identidad profesional, las narrativas y las acciones de respuesta y mediación a las sesiones estandarizadas en dos escuelas JEC. Asimismo, analiza el ejercicio de la autonomía profesional de los docentes como burócratas de primera línea en respuesta a una reforma impuesta de "arriba hacia abajo". El artículo proporciona evidencia sobre cómo las políticas educativas que son jerárquicas y verticales no reconocen la complejidad de la agencia de los actores escolares para mediar su implementación, lo que puede conducir a una erosión de la calidad de la educación en lugar de mejorarla (Ball, 1987, 1994, 2002; Braun et al. 2010; Comber y Nixon, 2009; Dale, 1992; Daviess, 1990; Day et al., 2006; Durose, 2017; Hohmann, 2016; Lipsky, 1980; Pollard et al., 1994; Sachs, 2003; Schweisfurth, 2002).

\section{Marco teórico}

\section{Reforma educativa bajo la agenda neoliberal}

El término "reforma educativa" se puede rastrear a los discursos y prácticas de los gobiernos neoliberales de los Estados Unidos y el Reino Unido en la década

5. Esta información fue obtenida a través de entrevistas a profundidad con los técnicos del Minedu, que estuvieron a cargo de la implementación de la reforma JEC en los años 2015 y 2016.

6. El término remite a la rendición de cuentas, que refiere al proceso en el que se vigila y evalúa el actuar responsable de los servidores públicos por medio de mecanismos como el monitoreo, la transparencia y la fiscalización. 
de 1980 (Campbell, 1996; Endacott et al., 2015; Gitlin y Margonis, 1995). Esta década marcó una nueva forma de hacer políticas educativas desde la influencia de las agendas neoliberales y mandatos economicistas, los que vinculan la reforma a la calidad, eficiencia y efectividad de los servicios escolares. Desde entonces, la expansión de las reformas neoliberales en educación ha adoptado una escala global dado el impulso de las agencias internacionales (Angotti, 2017; Tausch, 2010).

El foco en la calidad, eficacia, y eficiencia de los servicios y del gasto público impulsan reformas centradas en un discurso gerencialista. Este discurso propone metas y acciones estandarizadas que permiten un control claro de la provisión del servicio, y enfocan sus resultados en la medición del grado de fidelización entre el diseño de las reformas y su implementación (Comber y Nixon, 2009; Fuller et al., 2013; Lofty, 2003; Stone-Johnson, 2014; Yulindrasari y Ujianti, 2018).

A su vez, las metas y acciones estandarizadas requieren de sistemas de monitoreo que permitan comprobar si se alcanzan los objetivos de las reformas, lo cual crea una cantidad cada vez mayor de sistemas de seguimiento y monitoreo (Barrett, 2005; Brooks, 2009; Greenway et al., 2013; Huber y Skedsmo, 2016; Valli et al., 2007). Así, se promueve una cultura de auditoría que reforma las prácticas y las prioridades del sistema escolar y sus actores (Gerwitz, 2002; Stone-Johnson, 2014; Taylor, 2007; Taylor y Kelly, 2006; Wilkings 2011; Wong, 2008).

Además, el discurso gerencialista de las políticas educativas suele cuestionar el desempeño de los actores escolares asumiendo que no cuentan con incentivos suficientes para ejercer su autonomía a favor de la calidad, eficacia y eficiencia de los servicios. La percepción de bajo desempeño de las escuelas y las presiones externas para mejorar la calidad de los servicios permiten una mayor intervención centralizada por parte de gobierno para ganar control y acelerar las reformas (Ball, 1987, 1994, 2012; Campbell, 1996; Davies, 1990; Endacott et al, 2015; Gitlin y Margonis, 1995; Madsen, 1994; Pollard et al., 1994). Algunos estudios también señalan que el diseño de políticas jerárquicas y de solución única generalmente no se ajustan a la compleja realidad de las escuelas y aulas, lo cual genera contradicciones y socava la capacidad real del sistema para mejorar o reformarse (Campbell, 1996; Gitlin y Margonis, 1995; Katsuno,2012; Murphy y Skillen, 2015).

\section{Reformas jerárquicas y escuelas performativas}

Las reformas educativas bajo el enfoque gerencialista usualmente se definen como reformas de arriba hacia abajo (top-down) o reformas jerárquicas. Estas se caracterizan por ser cambios inducidos en los sistemas de manera vertical y jerarquizada, lo que asegura una disrupción en un estatus quo que es asumido como negativo o insuficiente. Estas características de la situación a reformar justifican la intervención y el control centralizado de políticas altamente 
estructuradas y con poco margen de adaptación (Berkovich, 2011; Hohmann, 2016; Taylor, 2007) ${ }^{7}$. Adicionalmente, este tipo de reformas educativas suelen inducir una estricta división de roles entre quienes definen las políticas (policy makers), y los que se encargan de implementarlas (policy delivers) y que deben ser evaluados por su desempeño (performance) (Ball, 1994; Bourke et al., 2015; Freidson, 2001).

La visión performativa de las reformas educativas (Hall y Noyes, 2009; Wilkings, 2015) suele representar a los docentes como ejecutores (delivers) que siguen e implementan indicaciones externas planteadas por los hacedores de política, en lugar de profesionales con juicio y criterio propio (Hall y McGinity, 2015; Lofty, 2003; Yulindrasari y Ujianti, 2018). Algunos de estos estudios proponen que, bajo estas premisas, la escuela actual se centra sobre todo en lograr la consecución de objetivos impuestos por agentes externos y vinculados a sistemas de rendición de cuentas, financiación y validación social que no necesariamente atienden a sus situaciones de contexto (Brooks, 2009; Brown y Manktelow, 2016; Lofty, 2003; Stone-Johnson, 2014; Webb, 2005).

Además, la visión performativa de la escuela entiende el rol del docente como un insumo (input) para el logro del resultado calidad educativa (outcome) mediada usualmente por los resultados de aprendizaje en pruebas estandarizadas para facilitar el seguimiento y la comparación de desempeño $(\text { output })^{8}$. Sin embargo, cuando los resultados de aprendizaje son bajos, el discurso político tiende a poner la responsabilidad en los profesores, cuestionando su profesionalismo y capacidad, a pesar de que estos no cuentan con la autonomía o recursos necesarios para poder hacerse totalmente responsables de los resultados de su labor (Greenway et al., 2013; Lofty, 2003; Mockler, 2011; Webb, 2005). Este tipo de cuestionamientos genera desconfianza en el juicio de los profesores, y así promueve una mayor estandarización de políticas y sistemas de vigilancia de los resultados escolares. Estos sistemas crean un círculo viscoso de desconfianza y centralización de las decisiones que erosiona la agencia y el profesionalismo docente, y refuerza las reformas jerarquizadas (Hult y Edström, 2016).

7. En contraposición se encuentran las reformas de abajo hacia arriba (bottom-up), las que son impulsadas por cambios sistemáticos que surgen desde dentro de las prácticas y la cultura escolar como resultado de procesos de adaptación, innovación o diseño de mejora intencionado (Davies, 1990; Marsh y Bowman, 1988; Sachs, 2001, 2003).

8. Dado que los resultados educativos como las trayectorias de vida de los estudiantes o la formación de socioemocional o ciudadanía son difíciles de medir y comparar, la educación suele centrarse en producir resultados de aprendizaje basados en pruebas estandarizadas nacionales e internacionales que se utilizan comúnmente como para el éxito de los sistemas educativos (Addison y McGee, 2015; Hult y Edström, 2016). Este tipo de evaluación puede conllevar a prácticas negativas, como entrenamientos para los exámenes, reducción del currículo o negligencia a los problemas que no son evaluados por el sistema (Braun et al., 2010; Gerwitz, 2012; Hall y Noyes, 2009; Webb, 2005; Wilkings, 2015). 
Los límites de la implementación de reformas jerárquicas: los arreglos institucionales y la agencia profesional

Las reformas jerárquicas imponen fines y prioridades de los sistemas educativos, en los que ya existen complejos arreglos institucionales, culturas, identidades, valores y prácticas instaladas que generan resistencias (Ball, 1987, 2002; Madsen, 1994; Murphy y Skillen, 2015). Esta imposición produce, además, cambios en los equilibrios de poder existentes, que redefinen las relaciones e interacciones, y pueden crear fricción y aislamiento. Por ejemplo, algunos estudios revelan cambios en la relación entre directivos y docentes, que, de una relación horizontal, se convierte en una relación jerárquica debido a los nuevos roles de responsabilidad y evaluación del personal de los administradores (Ball, 1994; Madsen, 1994; Sandfort, 2018). Asimismo, se han estudiado cambios en el papel de los padres en los sistemas educativos - los que pasan a asumir roles de consumidores o agentes de vigilancia (Karlsen Baeck, 2010)—, y en la forma de interacción y relación de los docentes. En este último caso, la colaboración y las agendas colectivas son reemplazadas por expresiones de individualismo, desconfianza y competitividad (Bosher y Jennings, 2005; Bourke et. al, 2015; Campbell, 1996; Valli et al., 2007).

Además de los cambios y resistencias en los arreglos institucionales, los actores educativos ejercen su agencia profesional y personal para mediar en la implementación de las políticas jerárquicas, lo cual abre una brecha entre la política en el discurso o papel, y la política ejecutada en la realidad (Braun et al., 2010; Dale, 1992; Durose, 2017; Easton, 1965; Hall y McGinity, 2015; Hohmann, 2016; Lee et al., 2013; Tang, 2011). Así, debido a la posibilidad de ejercer su agencia, los directivos y los docentes pueden tomar un rol de aceleradores, mediadores o saboteadores en la ruta de ejecución real de las reformas (Broodkin, 2008; Vähäsantanen et al., 2008).

Estos enfoques sobre la agencia y autonomía profesional en el marco de la implementación de políticas se basan en teoría fundacional de la "burocracia de la calle", desarrollada por Michael Lipsky. Según Lipsky:

La burocracia de la calle ejerce una amplia discreción en las decisiones finales que implementa para atender a los ciudadanos con quienes interactúan. Por ello, sus acciones individuales determinan el comportamiento de sus instituciones... Los roles de intermediación de políticas de los burócratas de la calle se basan en dos características interrelacionadas de su rol profesional: altos grados de discreción profesional y, alta autonomía relativa en su día a día. (1980 p. 13)

Esta mediación se asume como positiva para el sistema, porque permite a cada burócrata de la calle realizar adaptaciones o cambios que contextualicen la política para los requisitos de los usuarios reales con los que interactúa. Sin embargo, esto solo ocurre cuando los arreglos institucionales, la ética 
profesional y la provisión de recursos son los adecuados. Así, cuando los burócratas de la calle enfrentan malas condiciones de trabajo; recursos crónicamente inadecuados; o expectativas de metas ambiguas, vagas o contradictorias, pueden mostrar actitudes de agencia profesional negativa, como el absentismo, la alienación o la apatía (Lipsky, 1980, p.27).

La teoría de Lipsky sobre la burocracia de la calle permite comprender cómo la autonomía y la discreción profesional de los docentes se ven reducidas en el contexto de la implementación de las reformas educativas jerárquicas. Como efecto de esta reducción, la agencia profesional se erosiona y los docentes pierden la capacidad de mediar las reformas, incluso de manera positiva para adaptar los diseños únicos a la compleja realidad de su contexto (Barberis y Buchowitz, 2015; Bourke et al., 2015; Clabaugh, 2010; Hohmann, 2016; Mausethagen y Granlund, 2012; Taylor, 2007; Tummer y Bekker, 2014; Wong, 2008). Estos cambios entran en tensión con la forma en que los docentes solían comprender y experimentar su agencia, lo que genera respuestas hacia las reformas que pueden abarcar acciones que van desde el cumplimiento resignado a la resistencia activa (Berkovich, 2011; Braun et al.,2010; Dale, 1992; Gitlin y Margonis, 1995; Pollard et al., 1994).

Además, las reformas jerárquicas crean intensificación del trabajo, saturación de tiempo y desequilibrios de recursos, así como nuevas demandas que cumplir y múltiples prioridades impuestas, las que pueden promover la apatía, la alienación y el escepticismo de los actores educativos (Campbell, 1996; Hjörne et al, 2010; Murphy y Skillen, 2015; Sanford, 2018). Estas reacciones se agravan cuando se intensifica la presión externa por el rendimiento a través de los sistemas de accountability y monitoreo estandarizado (Benveniste, 1986; Boote, 2016; Brooks, 2009; Hjörne et al., 2010; Taylor y Kelly, 2006; Stone-Johnson, 2014; Webb, 2005).

\section{Agencias retadas y efectos en la identidad profesional docente}

En las reformas jerárquicas, la estandarización de objetivos educativos y, con ello, la implementación de prácticas en las que el personal especializado define externamente las estrategias y los materiales a ser usados en las aulas reducen a los docentes al rol de "semiprofesionales" (Comber y Nixon, 2009; Durose, 2017; Huber y Skedsmo,2016; Lofty, 2003; Thomas, 2011; Yulindrasari y Ujianti, 2018). La desprofesionalización se produce como consecuencia de la desconfianza en el criterio del docente, y es palpable en la intensificación de la jerarquía, los controles, la vigilancia de su desempeño y la reducción de su autonomía profesional (Broodkin, 2008; Freidson, 2001; Hult y Edström, 2016; Peters y Besley, 2015). Esta puede generar respuestas negativas y resistencia que afectan los resultados de las reformas y la calidad general del servicio educativo (Durose, 2017; Harrits y Østergaard, 2014; Marrón y Manktelow, 2016; Moore y Clarke, 2016; Ringwalt et al., 2008; Sandfort, 2018; Tummer y Bekkers, 2014). 
Estos cambios en la percepción externa sobre el rol profesional afectan también la identidad de los docentes?. Las reformas jerárquicas pueden tener un impacto negativo en su identidad profesional al desmoralizar o marginar a los docentes como efecto de los cambios en su autopercepción de su rol profesional como efecto de la reducción de autonomía y discreción, y del aumento de estandarización y vigilancia externa impuesta a su trabajo (Ebdacoot et al., 2015; Katsuno, 2012; Hall y Noyes, 2009; Wilkings, 2011).

Además, las identidades profesionales suelen afianzarse en valores y visiones del mundo desde los que se generan las respuestas de los docentes hacia la implementación de las reformas (Day et al. 2006; Lasky, 2005; Masoumpanah y Zarei, 2014). En ese sentido, cuando las identidades profesionales entran en conflicto con las reformas jerárquicas, dicha tensión puede promover acciones para preservar la autonomía, lo que genera respuestas contradictorias y contestatarias ante las reformas (Schweisfurth, 2002; Van Veen y Sleegers, 2006), y posturas políticas radicales y de resistencia extrema al cambio (Dale, 1992; Vähäsantanen et al., 2008).

\section{Metodología de investigación}

Este artículo se basa en un estudio exploratorio que emplea un muestreo de caso múltiple en dos escuelas secundaria que implementan el modelo. El diseño del estudio utilizó una selección de casos múltiple para comparar dos escuelas seleccionadas de manera intencionada y no aleatoria (Cohen et al., 2011, p. 291-292). Las dos escuelas JEC comparten condiciones similares sobre ubicación, número de aulas, número de docentes, matrícula y condición socioeconómica de las familias.

\section{Selección de casos}

El proceso de selección de casos se basó en los siguientes criterios:

- El universo escolar fue de 1601 escuelas que iniciaron la reforma JEC en 2015 y 2016.

9. La identidad profesional docente es entendida en la literatura como la forma en que los profesores se definen a sí mismos como profesionales de la educación (Beijaard et al., 2004; Carlyon, 2016; Lasky, 2005; Mockler, 2011). Las identidades son complejas y dinámicas, y pueden involucrar a los individuos y a sus roles y acciones colectivas, a los valores y la ética detrás de ellos, y a los intereses y posiciones de política (Tang, 2011; Vähäsantanen et al., 2008). También, incluye los factores profesionales - como los antecedentes educativos y la identificación con los sindicatos- y factores externos, que abarcan las condiciones de trabajo, el estado de la profesión y su valoración en la sociedad (Lee et al. 2013). Esta definición fusiona las visiones de la identidad docente individual y las visiones de la identidad docente como miembros de un colectivo o comunidad (Cohen, 2008; Vähäsantanen et. al., 2015). 
- Sobre la base de los resultados de la ECE, se seleccionó una submuestra de escuelas. Este proceso redujo el universo a veinte escuelas, diez de ellas con mejor desempeño nacional y otras diez con el peor desempeño. Dicha selección tuvo como objetivo generar dos casos similares con resultado diferente.

- Posteriormente, y debido a la diversidad del contexto de las escuelas en la submuestra, los casos potenciales se separaron por región, tipo de administración, y contexto rural o urbano.

- Las escuelas se emparejaron según la ubicación regional y las características de la escuela para tratar de aislar la variable de resultado en la prueba ECE.

- Las dos escuelas seleccionadas fueron las que mostraron el par más similar de condiciones y contexto, cuyos resultados solo difieren de aquellos obtenidos en la prueba ECE 2016.

Las escuelas seleccionadas están ubicadas en centros poblados de ruralidad no dispersa en la región sur andina del Perú, específicamente en las regiones de Apurímac y Puno. En el estudio, participaron un total de dieciséis docentes; solo un docente de una de las escuelas decidió no formar parte del estudio. Las características principales del grupo entrevistado de presentan en la siguiente tabla.

Tabla 1

Docentes en el estudio

\begin{tabular}{cccccc}
\hline Contratados & Nombrados & Varones & Mujeres & $\begin{array}{c}\text { Menores de } \\
35 \text { años }\end{array}$ & $\begin{array}{c}\text { Mayores de } \\
35 \text { años }\end{array}$ \\
\hline 8 & 8 & 11 & 5 & 6 & 10 \\
\hline
\end{tabular}

Métodos y recojo de información

La recolección de data priorizó el recojo de información de primera fuente mediante encuestas, entrevistas a profundidad y observación participante de las dinámicas escolares, tanto en las aulas como en los espacios cotidianos de la jornada escolar: formación, recreos, refrigerios y reuniones de docentes. La recolección de datos se realizó entre el mes de junio y la primera semana de julio de 2018. Durante ese período, se levantaron un total de dieciséis encuestas, diez entrevistas a profundidad y se recopilaron cuarenta páginas de notas de observación.

Se incluyen tres fuentes primarias en función de tres métodos de recojo complementarios:

- Encuestas exploratorias de veintidós preguntas para que los docentes informen sobre sus perfiles personales y profesionales, reflejen sus opiniones 
sobre la reforma de la secundaria JEC, así como su opinión y relación de uso con los materiales y sesiones estandarizadas.

- Entrevistas semiestructuradas de treinta minutos llevadas a cabo de manera individual con cada docente, y que estuvieron enfocadas en la profundización, obtención de ejemplos y un mayor desarrollo de las respuestas de la encuesta.

- Observaciones no estructuradas de la dinámica escolar y del aula.

Para mantener el sentido exploratorio de este recojo de información, se visitaron los casos sin un modelo teórico previo, o hipótesis sobre la mediación o acciones de los profesores (Bhatti, 2012).

Los permisos para realizar la investigación fueron otorgados por los directores y los docentes de las escuelas antes de la recopilación de datos. Además, los participantes fueron informados sobre el objetivo, el alcance y el uso de los datos proporcionados en una reunión general, así como de manera individual antes de la grabación de cada entrevista. Todos los entrevistados proporcionaron un consentimiento firmado para autorizar la encuesta, la entrevista, la grabación y el uso anónimo de sus declaraciones. Estas actividades siguieron todos los procedimientos de ética determinados por la Universidad de Glasgow para la investigación con actores escolares. En ese sentido, todos los datos recopilados se anonimizaron y codificaron para garantizar el derecho de los participantes a no ser reconocidos.

La metodología seleccionada y el diseño del estudio enfrentan la limitación de proporcionar información anecdótica y profunda sobre las dos escuelas seleccionadas. Las respuestas y los hallazgos informados como resultado de esta investigación no pueden generalizarse a otros docentes o entornos escolares en el Perú. Sin embargo, los hallazgos y su discusión pueden abrir más oportunidades para estudiar las respuestas de los profesores ante las reformas en educación y su mediación en la implementación de políticas a mayor escala. En ese sentido, este estudio proporciona información valiosa que puede ayudar a comprender el papel de la agencia docente en la implementación de reformas educativas en entornos con baja capacidad institucional estatal y condiciones precarias de trabajo.

\section{Hallazgos}

\section{Autonomía y discreción en un contexto de estandarización y vigilancia}

El ejercicio de la agencia profesional fue empleado por los docentes para mediar la implementación real de las sesiones estandarizadas entregadas por el Minedu. Los docentes implementaron estos materiales en sus aulas bajos sus propios términos, puesto que se consideran facultados para ejercer su autonomía y discreción, aun cuando existen presiones y demandas externas, princi66 I palmente relacionadas con el monitoreo. 
Según las respuestas de la encuesta, solo cuatro docentes (25\%) refirieron usar el paquete estandarizado siguiéndolo sin ninguna adaptación. Diez maestros $(62,5 \%)$ declararon que utilizaron sus propias planificaciones, incluidos los materiales estandarizados del Minedu solo cuando sentían que eran útiles. Dos (12,5\%) docentes no respondieron a esta pregunta. Además, cuando se preguntó a los docentes si se sentían autorizados a realizar adaptaciones en los materiales estandarizados, el 100\% respondió afirmativamente. En sus respuestas, dieron como razones las opciones 1 y 2 de la encuesta: "como profesional me siento con derecho a hacer cambios" y "si no se ajustan a la realidad de mi salón de clases, es mi prerrogativa decidir cómo uso el material", respectivamente.

Como se evidencia, los docentes que participaron del estudio no dudaron sobre si tenían o no derecho a ejercer su autonomía y discreción para mediar la implementación de la reforma a través del uso de las sesiones estandarizadas. Estos resultados muestran que se sienten empoderados en su rol profesional y agencia crítica. Algunas citas ayudan a evidenciar esta situación. Por ejemplo, uno de los docentes señala: "El Minedu no puede pedirnos que usemos los materiales como ellos quieren. Nosotros, como profesionales (sic), los profesores somos autónomos y debemos serlo siempre" (Docente 1, Caso 1). Otro de ellos afirma:

Siento que, si hice cambios en el material para adaptarlo a mi realidad, eso no puede ser algo equivocado (sic). Estoy mejorando el material. Ahí no hay ningún ejemplo de mi región. Si lo enseño así nomás, les diría que no es relevante nuestro pueblo. (Docente 6, Caso 2)

Además, los docentes declararon que el ejercicio de la autonomía y el uso de la discreción de su criterio profesional en el aula son deberes fundamentales de su profesión. Los profesores del estudio afirmaron que, al ejercerlos, están asegurando mejorar sus sesiones, y con ello el aprendizaje de los estudiantes a su cargo. Algunos de sus discursos ejemplifican estas ideas:

Hice cambios en el material proporcionado por el Minedu. Si yo no hago eso, mis alumnos no aprenderán. Tengo que hacer adaptaciones para mi salón de clases todo el tiempo. Eso, pues. Yo no puedo permitir que no aprendan. (Docente 4, Caso 2)

En esa misma línea, otro docente señala: "Por supuesto, siempre pues hacemos cambios en esas planificaciones. Es porque, de lo contrario, mis chicos no aprenderían. Quizás, sí se puedan usar sin cambio en un colegio grande, urbano" (Docente 3, Caso 1).

Los docentes declararon que la reforma JEC había desafiado su autonomía y discreción profesional. En ese sentido, sintieron que la existencia de materiales y sesiones estandarizados habilitaban al Minedu a ejercer mayor control 
sobre su trabajo, y que creían que los materiales se proporcionaron porque se desconfiaba de su trabajo y de su capacidad profesional.

Los docentes declararon que cada vez sentían menos libertad en el desarrollo de su trabajo, dada la provisión de normas altamente estandarizadas sobre las acciones del año escolar, a lo que se sumaba ahora materiales, sesiones y sistemas de monitoreo intensivo, y visitas no opinadas. Todas estas acciones alimentan una percepción negativa de las reformas dada la sensación de encontrarse en un ambiente agresivo de vigilancia, que afecta su desempeño y su autovaloración profesional. Algunas de las entrevistas reflejan estos discursos. Por ejemplo, un docente señaló: "Lo que me entristece un poco es que el Minedu nos obliga a usar los libros y, con ello, las sesiones. Siento que he perdido mi libertad para enseñar y elegir qué es lo mejor para mi clase" (Docente 2, Caso 1). Otro de los entrevistados añade: "Los monitores del Minedu vienen a observar nuestro trabajo. Pero no es para algo bueno. Es evaluarnos como si fuéramos malos siempre. Me siento vigilado" (Docente 4, Caso 1). Un tercer docente indica:

El Minedu quiere controlarnos; eso me hace sentir observado. No nos muestran cómo implementar esta reforma o capacitan, no más dan guías y guías, y se centran en exigirnos que hagamos lo que ellos quieren. Ahora deciden por nosotros, hasta nuestra nueva hora de salida la decidieron ellos, sin nosotros. (Docente 4, Caso 2)

Los docentes entrevistados enfrentan también demandas poco claras sobre el uso de las sesiones en el marco de la reforma JEC, lo que hace que desconfíen de sus decisiones sobre el uso del material estandarizado, al sentir cuestionadas sus acciones y criterios profesionales. En ese sentido, las respuestas de los profesores evidenciaron la poca claridad que existe sobre el uso del material identificando contradicciones. Mientras que en orientaciones y conferencias en línea se les anima a adaptar los materiales, los monitores, acompañantes y otros actores que llegan a sus aulas les exigen el uso de manera formal o implícita. Así, lo plantean los docentes entrevistados. Uno de ellos indica: "El monitor que viene quiere que nos alineemos con los materiales y las demandas del Ministerio. Me dicen: para eso les dimos el material" (Docente 1, Caso 2). En esa misma línea, otro señala: "En el Minedu siempre querrán que usemos el material. Pero no es posible hacerlo debido a las restricciones de tiempo y la realidad de mi aula, pero no quiero meterme en problemas. No está claro qué debería hacer" (Docente 5, Caso 2).

Además, los docentes afirmaron que las prácticas de seguimiento se perciben no solo como una acción de desconfianza, sino también como acciones que los subestiman y disminuyen como profesionales, lo que los hace sentir desanimados o enojados. 
Cuando llega la monitora del semáforo, entra a clase y me pregunta: “¿Usted usa los materiales?" y le digo que sí. Al ratito, les pregunta a unos alumnos: “¿Realmente usan los materiales? ¿Me muestras en qué página van?”. Me sentí avergonzado. (Docente 7, Caso 1)

Los docentes se refirieron a las visitas inopinadas o sorpresa de los monitores de Semáforo Escuela y monitoreo de prácticas pedagógicas como un seguimiento innecesario, y que se basa en un nivel de desconfianza percibido como injusto y abusivo. En respuesta, algunos han generado estrategias para mostrar aquello que asumen el monitoreo quiere ver, en lugar de cambiar a profundidad sus prácticas para alinearse a los objetivos de la reforma. Como señaló un docente durante la entrevista, "A veces, cuando vienen los monitores, le pedimos al alumno que traiga el libro, y practicamos cómo deben responder a las preguntas del monitor" (Docente 4, Caso 2). Otro docente se refirió a estas estrategias como "una forma de mantener una buena relación con el Minedu y las UGEL, y evitar meterse en problemas" (Docente 1, Caso 1).

Sin embargo, fuera del discurso de los docentes, las acciones del día a día no se alineaban a esta autopercepción de profesionalidad que no debería, según sus propios imaginarios, ser objeto de desconfianza por parte del Minedu. Durante las visitas escolares realizadas para la recolección de datos, que duró una semana y media en cada escuela, las clases se interrumpían sin motivo y de manera frecuente. El día escolar fue cancelado a la hora del recreo, en ambas escuelas, al menos dos veces por semana, sea por aniversarios, fiestas de la comunidad y otras actividades no pedagógicas, tales como practicar la marcha para fiestas patrias, partidos del mundial de fútbol y reuniones de docentes programadas en horas de clase. Los docentes también manejan discursos críticos sobre sus condiciones laborales para justificar las prácticas que pueden representarlos como poco profesionales; de esta manera, reivindican sus visiones positivas de su autonomía profesional. Esta situación genera una capa adicional interesante a su identidad profesional declarada que se contrapone a sus acciones, buscando justificaciones externas.

Estas contradicciones entre los discursos y las acciones reales son un punto interesante de entender para integrar el rol de la agencia docente en las reformas, sincerando sus dimensiones y complejidades.

\section{Reformas jerárquicas, condiciones laborales y profesionalismos retados: nosotros versus el Minedu}

Según las respuestas de la encuesta, el 90\% de los docentes de este estudio no se identifican con las razones e ideas que impulsaron la reforma, y sospechan que el Minedu está llevando a cabo estos cambios de manera apresurada y para desacreditar su desempeño, controlarlos y reducir su autonomía. En esa línea, algunos maestros declararon que creían que la reforma JEC era "un truco" para despedirlos y poder privatizar la educación pública. Estos discursos fueron 
recurrentes entre los profesores en el estudio, dado que, al tiempo que se realizaba la recopilación de datos, las bases del Sutep movilizaban el inicio de una nueva huelga nacional ${ }^{10}$ que no se llegó a concretar.

Las resistencias y los discursos que cuestionan la reforma se dan no solo debido al reto percibido en su agencia profesional y libertad de trabajo en aula, sino también por el gran cambio que la reforma JEC ha generado en sus vidas. Tener que asumir una jornada laboral extendida, que implica que deban quedarse en la escuela hasta las 3:15 pm, impide a los docentes tener un segundo trabajo o los obliga a vivir cerca de la escuela por la falta de transporte. Estos factores suman cuestionamiento y distanciamiento de la reforma JEC.

Además, las sesiones preparadas por el Minedu cambian la forma de enseñar y requieren interacciones frecuentes y sesiones activas que distan de las clases basadas en contenidos, comunes aún en la secundaria, y que no fueron cuestionadas por los docentes del estudio como un tema a mejorar o transformar. De acuerdo con la percepción de los docentes, la calidad educativa se relaciona, más bien, con la infraestructura; el servicio de alimentación; e, incluso, con el sueldo que perciben, antes que con el cambio de prácticas pedagógicas. Este distanciamiento con las finalidades de la reforma JEC impulsó a los docentes a contraatacar o ignorar los cambios demandados por el Minedu en sus acciones diarias.

En ese marco, los docentes también tienden a utilizar discursos que muestran desconfianza sobre el juicio y la capacidad del Minedu. Dicha desconfianza se basa en una percibida falta de adecuación entre el diseño de la reforma JEC, y los retos y complejidades que enfrentan estas escuelas rurales, lo que da pie a señalar que los funcionarios no conocen el terreno y que sus declaraciones públicas no muestran la realidad. Así, lo expresa uno de los docentes: "El Minedu les dirá que han mejorado la educación secundaria con JEC. Eso es una mentira. ¿Crees que esta escuela se parece a lo que dicen en el papel o lo que te cuentan en televisión?" (Docente 3, Caso 1). Ello proporciona una justificación razonable para resistir a los cambios y eludir a los monitores del Minedu, en tanto se percibe la reforma como no apta para el contexto escolar o como una medida que no necesita implementarse.

El imaginario sobre los especialistas del Minedu como "burócratas de cuello blanco" en contraposición de los profesores como "burócratas de la calle" refleja un tono de crítica sobre la poca conexión entre quienes diseñan las reformas y los contextos donde estas deben implementarse. Por ejemplo, uno de los docentes declaró: "Aquí los estudiantes caminan durante horas para llegar a la escuela. Ahora tienen quedarse hasta la tarde sin almorzar. Están cansados y el Minedu parece ignorar todo eso" (Docente 4, Caso 2). Otro de ellos señaló: "Será bueno para esta reforma que los funcionarios del Minedu JEC vengan a

10. En el mes de junio de 2018, se convocó a una nueva huelga magisterial, que generó un paro parcial en algunas escuelas, aunque no tuvo mucho éxito de convocatoria. 
visitar esta escuela. Ojalá vivieran en mis zapatos al menos por algunas horas" (Docente 6 - Caso 2).

En medio de desafiantes condiciones laborales, los profesores del estudio mantienen algunos discursos que reflejan sentimientos de menosprecio a su profesión por parte del Minedu. Estos sentimientos se han visto exacerbados por las políticas de estandarización y monitoreo, las que son percibidas como acciones claras de desconfianza y de desvalorización hacia su trabajo. Los docentes refirieron que no se sienten reconocidos, valorados o siquiera escuchados por el Minedu. Algunas citas de las entrevistas ilustran estas creencias. Uno de los entrevistados afirmó: "Hacemos cambios en los planes de lecciones. Mejoramos los materiales para adaptarse a nuestro contexto. Pero el Minedu no reconocerá eso. Para el Minedu, nosotros siempre vamos a ser menos que ellos" (Docente 1, Caso 2). Asimismo, uno indicó: "Solo unas pocas personas vinieron a esta escuela para escuchar lo que los maestros tienen que decir, sin juzgarnos, sin ya estar queriendo decir que los profesores somos malos" (Docente 4, Caso 1). Un tercer docente declaró: "El Minedu no nos valora. No son solo ellos. Para la sociedad, ser un maestro es el trabajo menos importante que alguien puede tener" (Docente 6, Caso 2).

\section{Discursos y acciones de los docentes en el contexto de la reforma de la educación secundaria en Perú}

Los docentes participantes del estudio promulgaron su agencia profesional para mediar la implementación de las sesiones estandarizadas también bajo retóricas contradictorias. Los discursos de los profesores muestran una evaluación positiva de las sesiones generadas por el Minedu que en otros momentos son criticadas duramente. Por ello, en la práctica, utilizan el material en su aula en diferentes medidas: algunos declararon que se sirven de sus propios materiales, mientras que otros optaron por adaptar las sesiones estandarizadas para que se ajusten a su contexto y a las necesidades de los estudiantes. Estos hallazgos dialogan con la literatura de la agencia docente y mediación del criterio profesional en el contexto de la implementación de la reforma (Berkovich, 2011; Endacott, et al., 2015; Schweisfurth, 2002).

Por su parte, las retóricas contradictorias de los docentes encajan en el concepto de imperativos competitivos, "situación en la que los profesores se encuentran atrapados entre diferentes versiones de cómo creen que actúan, cómo creen que deben actuar y cómo actúan en la realidad" (Schweisfurth, 2002, p. 63, citado en Alexander, 1995). Como efecto de estos imperativos, los discursos docentes y la forma en la que estos actúan pueden resultar incoherentes desde afuera.

Estos discursos y acciones sobre la reforma se pueden clasificar en un espectro de cinco estrategias: cumplimiento, incorporación, mediación, retraimiento y resistencia (Pollard et al., 1995, p. 100). No obstante, en este estudio, los discurso y acciones ante la reforma no encajan en una sola categoría, sino 
que pueden saltar de la incorporación a la resistencia rápidamente, o de la mediación al retraimiento, lo que se explica por la alta complejidad del contexto de estudio. La identidad profesional de los docentes, las condiciones laborales, las características de la reforma vertical y jerárquica podrían estar moldeando estas respuestas mixtas, lo que proporciona una entrada interesante para futuras investigaciones.

Los hallazgos también demuestran que los docentes se ven a sí mismos como profesionales autónomos y con discreción de implementación. En ese sentido, la reforma JEC se percibe como negativa, debido a que limita esta autonomía y discreción, y perjudica la autorregulación profesional de los docentes para remplazarlas por controles externos y verticales. Los discursos de los entrevistados sobre este tema critican la rendición de cuentas y el monitoreo, apelando al retorno de un sistema de confianza en el juicio profesional como forma de asegurar los resultados del aprendizaje. Estos hallazgos dialogan con los encontrados por otros estudios similares sobre implementación de reformas jerárquicas (Endacott et al., 2015; Hall y Noyes, 2009; Tang, 2011; Katsuno, 2012; Lee et al., 2013; Masoumpanah y Zarei, 2014; Schweisfurth, 2002; Vähäsantanen, 2015). Esta regularidad muestra que los desafíos sobre la identidad profesional y la agencia que generan las reformas verticales y jerárquicas se experimentan de manera similar por maestros sin importar el contexto.

Este estudio también evidencia que los docentes han normalizado sus prácticas no profesionales, como saltarse las horas de trabajo o engañar al sistema de rendición de cuentas. En un contexto de condiciones desafiantes, el estilo de implementación vertical de la reforma JEC, que implica demandas que se contradicen con la realidad que viven en aula, genera en los profesores la reacción de justificar sus acciones no profesionales sin asumir responsabilidad. Esto responde a que los vacíos de la reforma impuesta por el Minedu les proporciona excusas razonables para dudar, rechazar y resistir los cambios. El artículo abre una discusión sobre la agencia docente en un contexto de alta deprivación y desafíos a su profesionalismo. Este hallazgo dialoga con la noción de Lipsky sobre el "desapego de responsabilidad" de los burócratas de la calle:

Cuando las condiciones de trabajo reflejan recursos crónicamente inadecuados en relación con las tareas de los trabajadores deben cumplir y las expectativas de los objetivos de servicio tienden a ser ambiguas, vagas o mostrarse en conflicto, los burócratas de la calle pueden ejercer su autonomía y discreción para justificar prácticas negativas mediante el ejercicio de estrategias personales negativas como el absentismo, la alienación, y la apatía. (Lipsky, 1980 p. 27)

Finalmente, los hallazgos no mostraron ningún patrón en la respuesta con respecto a los perfiles del profesorado referidos a su edad, tipo de contrato, relación con el sindicato, años en la profesión, información que fue obtenida a partir de la encuesta aplicada en el estudio. Tampoco, se encontraron diferencias en las respuestas y los discursos entre la escuela con mejores resultados en la ECE y la escuela con los resultados más bajos. Este hecho desafía los hallaz72 I gos de otros estudios similares, que identificaron relaciones entre las respuestas 
hacia las reformas, los perfiles, los resultados de la escuela y las condiciones de trabajo (Carlyon, 2016; Lee et al., 2013; Ringwalt et al., 2003; Vähäsantanen et al., 2008).

\section{Conclusiones}

Este artículo está basado en una investigación exploratoria producto de un trabajo de campo a profundidad en dos escuelas secundarias rurales. Los hallazgos muestran que, en el contexto de una reforma de arriba hacia abajo - como la reforma JEC, que es acompañada de un sistema de accountability vertical para asegurar la implementación-, los docentes sienten desafiada su agencia profesional, y reaccionan mediante respuestas contradictorias y acciones de resistencia impulsadas por imperativos competitivos. Esto ocurre aun cuando los cambios que se busca introducir son coherentes con las reformas necesarias para mejorar la calidad educativa, como incluir sesiones modelo que rompan con la didáctica por contenidos aún vigente en la secundaria.

Los discursos docentes de imperativos competitivos, junto con el contexto de deprivación, reto y desconfianza, generan que los docentes vean los materiales estandarizados y las acciones de monitoreo como una amenaza a su agencia y discreción profesional. Sumado a ello, los vacíos en el diseño de la reforma y las demandas excesivas de monitoreo generan justificaciones para poner en marcha prácticas de mediación no positivas, que pueden alimentar las ideas de baja profesionalización y retiro de autonomía. Este hallazgo general dialoga con las conclusiones de estudios previos sobre el tema (Endacott et al., 2015; Hall y Noyes, 2009; Katsuno, 2012; Lee, 2013; Masoumpanah y Zarei, 2014; Tang, 2011; Teleshaliyev, 2013; Schweisfurth, 2002; Vähäsantanen, 2015; Wilkins, 2011).

La acción real de los docentes, observada por fuera de sus discursos, contribuye con la desconfianza del juicio profesional y la limitación de la autonomía para mejorar el ejercicio de la profesión docente. Los profesores reaccionan ante la desconfianza reforzando su propia comprensión del profesionalismo y sus valores colectivos, justificando sus prácticas poco profesionales. En ese escenario, se responsabiliza al Minedu y al contexto por las condiciones de la calidad educativa, y no se asume responsabilidad profesional propia, dado que los docentes se perciben con poca agencia para hacer los cambios que requiere el sistema.

Cuando los maestros asumen su agencia profesional de esta manera, responden a la reforma con una combinación compleja de "cumplimiento resistencia" y "mediación-retraimiento" (Pollard et al., 1995). Estas respuestas revelan que la efectividad de las reformas jerárquicas no se puede lograr únicamente con imposición y monitoreo, y que la autonomía profesional no siempre se defiende en favor de los estudiantes. Más estudios sobre la agencia del profesorado y su mediación en las reformas educativas son necesarios para comprender qué pasa en la última línea de la implementación de las políticas y 
embarcar la transformación real de las prácticas de los agentes educativos más importantes, los docentes, en el espíritu de emprender cambios que nos acerquen a un servicio educativo de calidad en el Perú. Asimismo, es importante indagar sobre los efectos de las reformas educativas jerárquicas reconociendo que las reformas de abajo hacia arriba recorren una ruta más pertinente a la compleja dinámica del cambio de prácticas por imposición, sobre todo, cuando expresan un cambio cultural a gran escala, pero que necesitan de políticas verticales que las nutran, sostengan y protejan de la poderosa inercia del sistema.

\section{Agradecimientos}

La autora hace un especial agradecimiento al Dr. Kristinn Hermannsson, profesor principal de la Universidad de Glasgow, Reino Unido, y supervisor de la investigación principal que da origen a este artículo. 


\section{Referencias}

Addison, J. y McGee, S. J. (2015). To the Core: College Composition Classrooms in the Age of Accountability, Standardized Testing, and Common Core State Standards. Rhetoric Review, 34(2), 200-218.

Alexander, R. (1995). Versions of Primary Education (1st ed.). Routledge.

Angotti T. (2017) Urban Latin America: Inequalities and Neoliberal Reforms. Rowman and Littlefield.

Ball, S. J. (1987). The Micro-Politics of the School: Toward a Theory of School Organization. Methuen. . (1994). Education Reform: A Critical and Post Structural Approach. Open University Press.

Barberis, E. y Buchowitz, I. (2015). Creating Accessibility to Education: The Role of School Staff's Discretionary Practices. European Education, 47(1), 61-76.

Barrett, A. M. (2005). Teacher Accountability in Context: Tanzanian Primary School Teachers' Perceptions of Local Community and Education Administration. Compare: A Journal of Comparative and International Education, 35(1), 43-61.

Beijaard, D., Meijer, P. C. y Verloop, N. (2004). Reconsidering Research on Teachers' Professional Identity. Teaching and Teacher Education, 20(2), 107-128.

Benveniste, G. (1986) School Accountability and the Professionalization of Teaching. Education and Urban Society, 18(3), 271-289.

Berkovich, I. (2011). No, We Won't! Teachers' Resistance to Educational Reform. Journal of Educational Administration, 49(1), 563-578.

Bhatti, G. (2017). Ethnographic Research. En: R. Coe, M. Waring, L. V. Hedges y J. Arthur, J (eds.), Research Methods and Methodologies in Education ( $2^{\text {nd }}$ ed, pp. 85-91). Sage.

Boote, D. N. (2016). Teachers Professional Discretion and the Curricula. Teachers and Teaching: theory and practice, 12(4), 461-478.

Bourke, T., Lidstone, J. y Ryan, M. (2013). Schooling Teachers: Professionalism or Disciplinary Power? Educational Philosophy and Theory, 47(1), 84-100.

Braun, A., Maguire, M. y Ball, S. J. (2010). Policy Enactments in the UK Secondary School: Examining Policy, Practice and School Positioning. Journal of Education Policy, 25(4), 547-560.

Broodkin, E. Z. (2008). Accountability in Street-Level Organizations Accountability in Street-Level Organizations. International Journal of Public Administration, 31(3), 317-336.

Brooks, C. (2009). Teaching in Full View: GLA as a Mechanism of Power. Policy Futures in Education, 7(3), 313-320. 
Brown, Z. y Manktelow, K. (2016). Perspectives on the Standards Agenda: Exploring the Agenda's Impact on Primary Teachers' Professional Identities. Education 3-13. International Journal of Primary, Elementary and Early Years Education, 44(1), 68-80.

Campbell, R. J. (1996). Educational Reform and Primary Teachers' Work: Some Sources of Conflict. Education 3-13. International Journal of Primary, Elementary and Early Years Education, 24(2), 13-22.

Carlyon, T. (2016). Teachers in Schools Transitioning from One-Year Level to Another: What Impact Does This Have on Teachers' Professional Identity?'. Asia-Pacific Journal of Teacher Education, 46(1), 98-110.

Clabaugh, G. K. (2010). The Cutting-Edge. Teacher Accountability and the Pathology of Domination. Educational Horizon, 88(4), 208-213.

Cohen, L. (2008). That's Not Treating You as A Professional': Teachers Constructing Complex Professional Identities Through Talk. Teachers and Teaching, 14(2), 79-93.

Manion, L. y Morrison, K. (2011). Research Methods in Education. Journal of Educational, Cultural and Psychological Studies (ECPS Journal), 2(4), 201-206.

Comber, B. y Nixon, H. (2009). Teachers' Work and Pedagogy in an Era of Accountability. Discourse Studies in the Cultural Politics of Education, 30(3), 333-345.

Davies, L. (1990). Equity and Efficiency: School Management in an International Context. The Falmer Press.

Day, C., Kington, A., Stobart, G., y Sammons, P. (2006). The Personal and Professional Selves of Teachers: Stable and Unstable Identities. British Educational Research Journal, 32(4), 601-616.

Durose, C. (2007). Beyond "Street Level Bureaucrats": Re-Interpreting the Role of Front-Line Public-Sector Workers. Critical Policy Studies, 1(2), 217234.

Endacott, J., Norton, G., Goering, C., Collet, V., Denny, G., y Davis, J. (2015). Robots Teaching Other Little Robots: Neoliberalism, CCSS, and Teacher Professionalism. Review of Education, Pedagogy, and Cultural Studies, 37(5), 414-437.

Easton, D. (2017). A Systems Analysis of Political Life. En: W. Buckley (Ed.), Systems Research for Behavioral Science Research. A Sourcebook (pp. 428436). Routledge.

Freidson, E. (2001). Professionalism: The Third Logic. Polity Press.

Fuller, C., Goodwyn, A. y Francis-Brophy, E. (2013). Advanced Skills Teachers: Professional Identity and Status. Teachers and Teaching: Theory and Practice, 19(4), 463-474.

Gewirtz, S. (2002). The Managerial School: Post-Welfarism and Social Justice in Education. Routledge. 
Gitlin, A. y Margonis, F. (1995). The Political Aspect of Reform: Teacher Resistance as Good Sense. American Journal of Education,103(4), 377405.

Greenway, R., McCollow, M., Hudson, R. F., Peck, C. y Davis, C. A. (2013). Division on Autism and Developmental Disabilities Autonomy and Accountability: Teacher Perspectives on Evidence-Based Practice and Decision-Making for Students with Intellectual and Developmental Disabilities. Education and Training in Autism and Developmental Disabilities, 48(484), 456-468.

Hall, C. y Noyes, A. (2009). New Regimes of Truth: The Impact of Performative School Self-Evaluation Systems on Teachers' Professional Identities. Teaching and Teacher Education, 25(6), 850-856.

Hall, D. y McGinity, R. (2015). Conceptualizing Teacher Professional Identity in Neoliberal Times: Resistance, Compliance and Reform. Education policy analysis archives, 23(88).

Harrits, G. S. y Møller Østergaard, M. (2014). Prevention at the Front Line: How Home Nurses, Pedagogues, and Teachers Transform Public Worry into Decisions on Special Efforts. Public Management Review, 16(4), 447480.

Hjörne, E., Juhila, K. y Nijnatten, C. Van (2010). Negotiating Dilemmas in the Practices of Street-Level Welfare Work 1. International Journal of Social Welfare, 19, 303-309.

Hohmann, U. (2016). Making Policy in the Classroom. Research in Comparative and International Education, 11(4), 380-393.

Huber, S. G. y Skedsmo, G. (2016). Teacher Evaluation - Accountability and Improving Teaching Practices. Educational Assessment, Evaluation and Accountability. Educational Assessment, Evaluation and Accountability, 28(1), 105-109.

Hult, A. y Edström, C. (2016). Teacher Ambivalence Towards School Evaluation: Promoting and Ruining Teacher Professionalism. Education Inquiry, 7(3), 305-325.

Karlsen Baeck, U.-D. (2010). "We Are the Professionals": A Study of Teachers' Views on Parental Involvement in School. Source: British Journal of Sociology of Education British Journal of Sociology of Education, 31(3), 323-335.

Katsuno, M. (2012). Teachers' Professional Identities in an Era of Testing Accountability in Japan: The Case of Teachers in Low-Performing Schools. Education Research International, 3.

Lasky, S. (2005). A Sociocultural Approach to Understanding Teacher Identity, Agency and Professional Vulnerability in A Context of Secondary School Reform. Teaching and Teacher Education, 21(8), 899-916. 
Lee, J., Xian-Han Huang J., Hau-Fai Law, E. y Wang, M. (2013). Professional Identities and Emotions of Teachers in the Context of Curriculum Reform: A Chinese Perspective. Asia-Pacific Journal of Teacher Education, 41(3), 271-287.

Lipsky, M. (1980). Street-Level Bureaucracy. Russell Sage Foundation.

Lofty, J. S. (2003). Standards and the Politics of Time and Teacher Professionalism. English Education, 35(3), 195-222.

Madsen, J. (1994). Educational Reform at the State Level. The Falmer Press.

Marsh, D. y Bowman, G. A. (1988). State-Initiated Top-Down Versus BottomUp Reform in Secondary Schools. National Center on Effective Secondary Schools, University of Wisconsin-Madison.

Masoumpanah, Z. y Zarei, G. R. (2014). EIL, Iranian Teachers' Professional Identity and Perception of Professional Competence. Procedia - Social and Behavioral Sciences.

Mausethagen, S. y Granlund, L. (2012). Contested Discourses of Teacher Professionalism: Current Tensions Between Education Policy and Teachers' Union, Journal of Education Policy, 27(6), 815-833.

Mockler, N. (2011). Beyond "What Works": Understanding Teacher Identity as a Practical and Political Tool. Teachers and Teaching: Theory and Practice Teachers and Teaching, 17(5), 517-528.

Moore, A. y Clarke, M. (2016). "Cruel optimism": Teacher Attachment to Professionalism in an Era of Performativity'. Journal of Education Policy, 31(5), 666-677.

Murphy, M. y Skillen, P. (2015). The Politics of Time on The Front Line: Street Level Bureaucracy, Professional Judgement and Public Accountability. International Journal of Public Administration, 38(August), 632-641.

OECD (2018). About PISA. http://www.oecd.org/pisa/aboutpisa/.

Pollard, A., Broadfoot, P., Croll, P., Osborn, M. y Abbott, D. (1994). Changing English Primary Schools? The Impact of the Educational Reform Act at Key Stage One. Cassel.

Resolución Ministerial N³01-2014-MINEDU: Creación del Grupo de Trabajo encargado de formular el modelo de servicio educativo: Jornada Escolar Completa para las Instituciones educativas públicas del Nivel de educación secundaria, el plan de implementación y las acciones de seguimiento del referido modelo. 11 de julio de 2014.

Resolución Ministerial No 451-2014-MINEDU: Crea el modelo de servicio educativo Jornada Escolar Completa para las Instituciones Educativas públicas del nivel de educación secundaria'. 1 de octubre de 2014.

Resolución Ministerial N 389-2015-MINEDU: Aprueban el listado de las 604 instituciones educativas en las cuales se implementará el modelo de servicio educativo "Jornada Escolar Completa para las instituciones 
educativas públicas del nivel de educación secundaria” durante el año 2016. 11 de agosto de 2015.

Ringwalt, C. L. (2003). Factors Associated with Fidelity to Substance Use Prevention Curriculum Guides in the Nation's Middle Schools. Health Education y Behavior, 30(3), 375-491.

Sachs, J. (2001). Teacher Professional Identity: Competing Discourses, Competing Outcomes'. Journal of Education Policy, 16(2), 149-161. . (2003). The Activist Teaching Profession. Open University Press.

Sandfort, J. R. (2018). Moving Beyond Discretion and Outcomes: Examining Public Management from the Front Lines of the Welfare System. Journal of Public Administration Research and Theory, 10(4), 729-756.

Schweisfurth, M. (2002). Democracy and Teacher Education: Negotiating Practice in The Gambia. Comparative Education. 38(3), 303-314.

Stone-Johnson, C. (2014). Parallel Professionalism in an Era of Standardization. Teachers and Teaching: Theory and Practice, 20(1), 74-91.

Tang, S. Y. F. (2011). Teachers' Professional Identity, Educational Change And Neo-Liberal Pressures on Education in Hong Kong, Teacher Development, 15(3), 363-380.

Tausch, A. (2010). Globalization and Development: The Relevance of Classical "Dependency" Theory for the World Today. International Social Science Journal, 61(202), 467-488.

Taylor, I. (2007). Discretion and Control in Education: The Teacher as Street-Level Bureaucrat. Educational Management Administration and Leadership, 35(4), 555-572.

Taylor, I. y Kelly, J. (2006). Professionals, Discretion And Public Sector Reform In The UK: Re-Visiting Lipsky'. International Journal of Public Sector Management, 19(7), 629-642.

Teleshaliyev, N. (2013). Leave Me Alone Simply Let Me Teach. European Education, 45(2), 51-74.

Tummers, L. y Bekkers, V. (2014). Policy Implementation, Street-level Bureaucracy, and the Importance of Discretion. Public Management Review, 16(4), 527-547.

Vähäsantanen, K. (2015). Professional Agency in the Stream of Change: Understanding Educational Change and Teachers' Professional Identities. Teaching and Teacher Education, 47, 1-12.

Vähäsantanen, K., Hökkä, P., Eteläpelto, A., Rasku-Puttonen, H., y Littleton, K. (2008). Teachers' Professional Identity Negotiations in Two Different Work Organisations. Vocations and Learning, 1(2).

Valli, L., Croninger, R. G. y Walters, K. (2007). Who (Else) Is the Teacher? Cautionary Notes on Teacher Accountability Systems. American Journal of Education, 113(4), 635-662. 
Van Veen, K. y Sleegers, P. (2006). How Does It Feel? Teachers' Emotions in a Context of Change. Journal of Curriculum Studies, 38(1), 85-111.

Webb, P. T. (2005). The Anatomy of Accountability. Journal of Education Policy, 20(2), 189-208.

Wilkins, C. (2011). Professionalism and the Post-Performative Teacher: New Teachers Reflect on Autonomy and Accountability in the English School System, Professional Development in Education, 37(3), 389-409. . (2015). Education reform in England: Quality and Equity in the Performative School. International Journal of Inclusive Education, 19(11), 1143-1160.

Wong, J. L. N. (2008). How Does the New Emphasis on Managerialism in Education Redefine Teacher Professionalism? A Case Study in Guangdong Province of China. Educational Review, 60(3), 267-282. “

Yulindrasari, H. y Ujianti, P. R. (2018). “Trapped in the Reform”: Kindergarten teachers' experiences of teacher professionalization in Buleleng, Indonesia. Policy Futures in Education, 16(1), 66-79. 